\title{
The presence of anaemia negatively influences survival in patients with POLG disease
}

Omar Hikmat $^{1,2}$, Tzoulis Charalampos ${ }^{2,3}$, Claus Klingenberg ${ }^{4,5}$, Magnhild Rasmussen ${ }^{6}$, Chantal ME Tallaksen ${ }^{7,8}$, Eylert Brodtkorb ${ }^{9,10}$,Torunn Fiskerstrand ${ }^{1,12}$, Robert McFarland ${ }^{13}$, Shamima Rahman ${ }^{14,15}$, Laurence A. Bindoff ${ }^{2,3}$

1. Department of Pediatrics, Haukeland University Hospital, 5021 Bergen, Norway.

2. Department of Clinical Medicine (K1), University of Bergen, Norway.

3. Department of Neurology, Haukeland University Hospital, 5021 Bergen, Norway

4. Department of Paediatric and Adolescent Medicine, University Hospital of North Norway, Troms $\varnothing$, Norway.

5. Paediatric Research Group, Department of Clinical Medicine, UiT- The Arctic University of Norway, Troms $\emptyset$, Norway.

6.Women and Children's Division, Department of Clinical Neurosciences for Children, Oslo University Hospital, Oslo, Norway and Unit for Congenital and Hereditary Neuromuscular Disorders, Department of Neurology, Oslo University Hospital, Oslo, Norway.

7. Department of Neurology, Oslo University Hospital, Oslo, Norway.

8. Institute of Clinical Medicine, Faculty of Medicine, University of Oslo, Oslo, Norway.

9. Department of Neuroscience, Norwegian University of Science and Technology,

Trondheim, Norway.

10. Department of Neurology and Clinical Neurophysiology, St. Olav's University Hospital, Trondheim, Norway.

11. Center for Medical Genetics and Molecular Medicine, Haukeland University Hospital, Bergen, Norway.

12. Department of Clinical Science (K2), University of Bergen, Norway. 
13. Wellcome Trust Centre for Mitochondrial Research, Institute of Neuroscience, The

Medical School Framlington Place, Newcastle University, Newcastle upon Tyne, UK.

14. Mitochondrial Research Group, UCL Great Ormond Street Institute of Child Health, London, UK.

15. Metabolic Unit, Great Ormond Street Hospital NHS Foundation trust, London, UK.

\section{Correspondence to:}

Professor Laurence Bindoff

Department of Neurology

University of Bergen

Haukeland University Hospital

5021 Bergen, Norway

Tel: +47 55975096

Fax: +47 55975164

e-mail:laurence.bindoff@ nevro.uib.no

Total number of words: 2150

Number of figures and tables: 2 tables and 2 figures. 


\section{Abstract \\ Background}

Mitochondria play an important role in iron metabolism and hematopoietic cell homeostasis. Recent studies in mice showed that a mutation in the catalytic subunit of polymerase gamma $(P O L G)$ was associated with hematopoietic dysfunction including anaemia. The aim of this study was to analyse the frequency of anaemia in a large cohort of patients with $P O L G$ related disease.

\section{Methods}

We conducted a multi-national, retrospective study of 61 patients with confirmed, pathogenic biallelic POLG mutations from six centres, four in Norway and two in the United Kingdom. Clinical, laboratory and genetic data were collected using a structured questionnaire. Anaemia was defined as an abnormally low haemoglobin value adjusted for age and sex. Univariate survival analysis was performed using log-rank test to compare differences in survival time between categories.

\section{Results}

Anaemia occurred in $67 \%(41 / 61)$ of patients and in $23 \%(14 / 61)$ it was already present at clinical presentation. The frequency of anaemia in patients with early onset disease including Alpers syndrome and myocerebrohepatopathy spectrum (MCHS) was high (72\%) and 35\% $(8 / 23)$ of these had anaemia at presentation. Survival analysis showed that the presence of anaemia was associated with a significantly worse survival $(P=0.004)$.

\section{Conclusion}

Our study reveals that anaemia can be a feature of $P O L G$-related disease. Further, we show that its presence is associated with significantly worse prognosis either because anaemia itself is impacting survival or because it reflects the presence of more serious disease. In either case, our data suggests anaemia is a marker for negative prognosis. 
Take-home message (synopsis): Anaemia is an important negative prognostic marker of $P O L G$ related disease and one that is associated with significantly worse survival.

\section{Details of the contributions of individual authors:}

Hikmat and Bindoff designed the study, and were responsible for data collection and analysis. They drafted the initial manuscript, approved the final manuscript as submitted, and were responsible for accuracy and integrity of the work.

Tzoulis, Klingenberg, Rasmussen, Tallaksen, Brodtkorb, Fiskerstrand, McFarland and Rahman were responsible for acquisition of clinical data, critically revising and approving the manuscript as submitted.

All authors are responsible of accuracy and integrity of the work

Disclosure of conflicts of interest: The authors declare no financial or other conflicts of interest.

Details of funding: This work was supported by grants from the Western Norway Regional Health Authority (Helse Vest, grants no.911944).

Details of ethics approval: All procedures followed were in accordance with the ethical standards of the responsible committee on human experimentation (institutional and national) and with the Helsinki Declaration of 1975, as revised in 2000 (5). The ethical approved for the study was obtained from the Regional Committee for Medical and Health Research Ethics, Western Norway (REK 2014/1783-4). The study was registered as an audit at Great Ormond Street Hospital, London, UK. Anonymised data regarding Newcastle patients was provided courtesy of the MRC Mitochondrial Disease Patient Cohort (Ethics ref:13/NE/0326) Keywords: Alpers, Anaemia, Hematopoietic dysfunction, Iron metabolism, Mitochondria, POLG. 


\section{Introduction}

Mitochondria play an important role in maintaining cellular homeostasis being important not only for energy metabolism and linked processes such as the tricarboxylic acid cycle and fatty acid oxidation, but also in iron-sulphur biogenesis, porphyrin and pyrimidine biosynthesis, calcium buffering and signalling and phospholipid metabolism (Lu and Claypool 2015). Moreover, mitochondria are considered to be the major producer of reactive oxygen species (ROS) which are directly involved in apoptosis (Green and Kroemer 2004) .

Mitochondrial dysfunction leads to a wide spectrum of well recognized human disorders with variable manifestations and age of onset. Haematological manifestations of mitochondrial dysfunction have been reported (Fontenay et al 2006; Camaschella 2009), but are considered uncommon. Pearson syndrome (OMIM 557000) is a sporadic mitochondrial disorder caused by large scale deletion of mitochondrial DNA (mtDNA); it presents in the first months of life with refractory sideroblastic anaemia, exocrine pancreatic dysfunction and multisystem involvement with liver failure and proximal tubulopathy. It was originally described in 1979 and was one of the first examples demonstrating a causal relationship between mitochondrial dysfunction and haematological disease (Pearson et al 1979; Rotig et al 1995). Since then, mutations in multiple mitochondrial genes involved in widely differing processes have been associated with haematological disease (Table1). (Antonicka et al 2003; Fontenay et al 2006; Morison et al 2008; Shteyer et al 2009; Ojala et al 2012; Clarke et al 2013; Hildick-Smith et al 2013; Burrage et al 2014; Schmitz-Abe et al 2015; Wortmann et al 2015; Riley et al 2016; Wedatilake et al 2016; Torraco et al 2017)

The integrity of mtDNA is critically dependent upon polymerase gamma (Pol $\gamma$ ) which is necessary for replication and repair of this genome. Mutations in POLG (MIN\#174763, POLG1) encoding the catalytic subunit of pol $\gamma$ manifest a wide spectrum of phenotypes that 
affect multiple organ systems including the central and peripheral nervous systems, skeletal muscle, liver, endocrine glands and the gut (Hudson and Chinnery 2006; Saneto and Naviaux 2010). The two major phenotypes associated with early onset $P O L G$ related disorders are Alpers syndrome and the myocerebrohepatopathy spectrum (MCHS). Alpers syndrome (OMIM \# 203700) is one of the most commonly reported phenotypes of POLG related disease in the paediatric population and is associated with high mortality (Nguyen et al 2006). Clinically, Alpers is characterized by a triad of progressive encephalopathy with psychomotor regression, refractory epilepsy and (frequently but not always) characteristic liver pathology while MCHS, another severe and fatal form of early onset $P O L G$ related disease, presents most frequently with a triad of myopathy or hypotonia, developmental delay or encephalopathy and liver dysfunction (Nguyen et al 2006) (Hikmat et al 2017 GiM in press). In the adult population, epilepsy and ataxia dominate the clinical picture (Winterthun et al 2005; Tzoulis et al 2006).

Haematological disorders associated with $P O L G$ mutation have not been reported. Recent studies showed, however, that an animal model carrying a proof reading deficient pol $\gamma$ manifested anaemia and haematopoietic progenitor dysfunction present from intrauterine life, erythroid dysplasia and impaired lymphopoiesis (Trifunovic et al 2004; Chen et al 2009; Ahlqvist et al 2012; Ahlqvist et al 2015; Li-Harms et al 2015).

The aim of this study was to investigate the occurrence of anaemia in a large cohort of patients with $P O L G$ related disease.

\section{Materials and methods}

We conducted a multi-national, descriptive, retrospective study in which six centres participated; Norwegian centres were: Haukeland University Hospital, Oslo University 
Hospital, St. Olav Hospital, Trondheim and University Hospital of North Norway; UK

Centres were: Great Ormond Street Hospital and Wellcome Trust Centre for Mitochondrial Research, Institute of Neuroscience, Newcastle University. Patients with confirmed pathogenic biallelic POLG mutations who had been diagnosed and followed up at the participating centres were considered eligible.

Clinical, laboratory and genetic data were collected using a structured questionnaire completed by a responsible investigator. The questionnaire was developed and tested in a pilot study conducted at Haukeland University Hospital on ten patients with confirmed pathogenic biallelic POLG mutations. The main cohort was divided into two groups according to the age of onset, with 16 years used as a cut off to enable analysis of anaemia in those with onset before puberty, including delayed puberty, and those with onset after.

All the patients were analysed for anaemia both at onset and during the course of the disease. Anaemia was defined as an abnormally low haemoglobin value according to age and sex adjust values (Nordin et al 2004)(Table 2). Anaemia due to blood loss was excluded. All genetic studies were performed with informed consent. The ethical approved for the study was obtained from the Regional Committee for Medical and Health Research Ethics, Western Norway (REK 2014/1783-4). The study was registered as an audit at Great Ormond Street Hospital, London, UK. Anonymised data regarding Newcastle patients was provided courtesy of the MRC Mitochondrial Disease Patient Cohort (Ethics ref:13/NE/0326).

\section{Statistical analysis}

Data were analyzed using SPSS (Statistical Package of Social Sciences), Version 22.0 (SPSS. Inc. Chicago, IL). A two sided $P$ value less than 0.05 was considered to be statistically 
significant. Categories were compared using Fisher`s Exact tests when appropriate. For survival analysis, the end-point was time to death which was defined as the time in months from the date of onset to the date of death. Univariate survival analysis was performed using log-rank test (Kaplan Meier) to compare differences in survival time between categories. For vector graphics, Adobe illustrator CS6 was used to edit the figure. 


\section{Results}

A total of 61 patients (30 males; 31 females) were enrolled. Median age at disease onset was 6.7 years (range 1 month -53 years) (Table 2). Forty-five patients were diagnosed in Norway and 16 in the UK. The majority $(n=57)$ of patients were North European, while two patients were from Cyprus and one from the Middle East and Pakistan. Seventy two percent (44/61) were aged less than 16 years at disease onset. More details concerning the phenotypes and genotypes are provided in supplementary file 1.

Anaemia was present in $67 \%(n=41 / 61)$ of patients at some point prior to the terminal stage of their disease. The age and sex related median haemoglobin values included in this study are summarized in table 2 . The presence of anaemia was similar in those manifesting before $(68 \%, n=30 / 44)$ or after 16 years $(65 \%, n=11 / 17)$, and was marginally more common in males $(70 \%, n=21 / 30)$ than females $(64 \%, n=20 / 31)$. Fourteen patients $(23 \%, n=14 / 61)$ had anaemia at clinical presentation of their disease, and only $35 \%$ of those $(n=5 / 14)$ had evidence of liver pathology. The frequency of anaemia in patients with early onset POLG disease (Alpers syndrome and MCHS phenotypes) was high $(72 \%, \mathrm{n}=18 / 25)$ and $35 \%(\mathrm{n}=$ 8/23) of these patients had anaemia at disease onset.

Limited data on the cause of anaemia was available in 12 patients; 10 had iron deficiency and 2 had low folate. In the remainder, insufficient data was available to classify the type of anaemia. Bone marrow aspirate was performed on one patient (homozygous for c.1399G>A; p.Ala467Thr) aged 47 years and showed ineffective erythropoiesis with no abnormalities in other elements of the bone marrow. 
In order to assess the potential impact of anaemia, we analysed survival according to haemoglobin status and the presence/absence of anaemia during disease course. The presence of anaemia was associated with a significantly worse survival $(P=0.004)$ (Figure1) and there was no significant difference in the survival between those manifesting anaemia at disease onset compared to those who developed anaemia during the course of their disease. There was no clear genotype-phenotype correlation with mutations in all regions of $P O L G$ associated with anaemia (Figure 2). 


\section{Discussion}

Our study is the first to show that anaemia is a feature of human $P O L G$-related mitochondrial disease. Further, we show that the presence of low haemoglobin signals a poor prognosis, irrespective of when it occurs.

Mitochondria play an important role in haematopoietic cell homeostasis and iron metabolism; the reversible oxidative state of iron enables the mitochondrial respiratory chain to catalyse electron transport through its haem and iron sulphur cluster containing proteins, and use this process in energy transduction (Richardson et al 2010). Mitochondria are also the major site of haem biosynthesis with four of the eight biosynthetic steps occurring within this organelle (Lill and Muhlenhoff 2008). Haematological disease due to mitochondrial dysfunction is nevertheless considered uncommon.

Mutations in $P O L G$ are currently considered the most common single gene cause of mitochondrial disease (Saneto and Naviaux 2010) with frequent involvement of organ systems such as the brain, muscle and liver. Little attention has been given to the haematological system. Recent studies performed in the POLG mutator mouse model, however, demonstrated that $P O L G$ mutation can also drive haematopoietic abnormalities including anaemia (Trifunovic et al 2004; Chen et al 2009; Ahlqvist et al 2012; Ahlqvist et al 2015; Li-Harms et al 2015). Similar changes, namely delayed maturation of erythrocytes that still contained mitochondria, were also seen in the mtDNA deletion disorder Pearson syndrome.

In the absence of data in humans, we analysed the frequency of anaemia in a large cohort of patients with genetically confirmed $P O L G$ mutations. These patients were ascertained from 6 
different centres and spanned the whole spectrum of $P O L G$-related disease ( supplementary file 1). Our findings confirm that mutated $P O L G$ is associated with anaemia in humans. Approximately two third of the patients enrolled in this study developed anaemia at some stage and importantly almost one in four $(23 \%)$ already had anaemia at presentation. This was particularly true for patients with early onset $P O L G$ related disease (Alpers syndrome and MCHS phenotypes) who had a high frequency of anaemia (72\%) with $35 \%$ having anaemia at presentation.

Anaemia can be caused by three independent mechanisms: decreased red blood cell (RBC) production, increased RBC destruction and blood loss. None of our patients had any clinical or laboratory evidence of haemolysis or blood loss at the time that anaemia was recorded. Those who were included in the cohort, who went on to develop terminal multi-organ failure already had anaemia registered at an earlier time point. This suggests that the cause of the anaemia was decreased $\mathrm{RBC}$ production. The presence of anaemia at disease presentation argues strongly against an "anaemia of chronic disease" or it being due to the influence of therapy e.g. anticonvulsant medications. The liver is the main organ of haematopoiesis from the third to the sixth intrauterine month and continues to produce RBC into the first postnatal week before the bone marrow takes over as the only site for blood cell production. While liver involvement is well recognised in POLG related disease, we found that only $35 \%(5 / 14)$ of those with anaemia at disease onset had manifest liver cell dysfunction; three had Alpers with only mild elevation of aspartate aminotransferase (ASAT) or alanine aminotransferase (ALAT), while two had the MCHS phenotype with clear evidence of liver failure. All were between 3-12 months of age. Our findings suggest, therefore, that decreased RBC production and not liver dysfunction is the cause of the anaemia. Animal studies also support this hypothesis showing that $P O L G$ mutation was associated with anaemia, haematopoietic 
progenitor dysfunction and erythroid dysplasia (Trifunovic et al 2004; Chen et al 2009; Ahlqvist et al 2012; Ahlqvist et al 2015; Li-Harms et al 2015).

In those patients in whom anaemia occurred later, i.e. after presentation, our study suggests that iron deficiency is the major cause. Since this was a retrospective review of patient notes in which data on nutritional and other metabolic abnormalities was patchy or unavailable, we are able to provide an incomplete picture of the causes of the anaemia found in this cohort. The only patient investigated with bone marrow aspirate did however, show ineffective erythropoiesis as the most likely cause of the anaemia.

Irrespective of cause, the presence of anaemia was associated with significantly worse survival whether it was present early or later in the disease course. What, therefore, is the link between anaemia and mitochondrial dysfunction? In a study using matched quantitative genomic and proteomic analysis in mouse muscle cells, iron deprivation resulted in a rapid, dose-dependent decrease of mitochondrial proteins and oxidative capacity that was fully reversed when iron was reintroduced (Rensvold et al 2013). Subsequent studies showed that the transcriptional changes were accompanied by alterations to histone acetylation and methylation levels that were largely reversible by reintroduction of iron (Rensvold et al 2016). We show that anaemia is associated with significantly worse prognosis, and that iron deficiency may be its cause. Based on these findings, one could ask whether we are missing a potential therapeutic opportunity by not treating the anaemia. Optimizing iron and haemoglobin levels may both improve survival and decrease the morbidity associated with $P O L G$ related disorders.

In conclusion, we show that anaemia is an important negative prognostic marker of $P O L G-$ related disease and one that is associated with significantly worse survival. Further 
prospective studies are needed to investigate whether optimization of haemoglobin and iron levels improve survival and decrease morbidity associated with $P O L G$ mutations. 


\section{Acknowledgements}

This work was supported by grants from the Western Norway Regional Health Authority (Helse Vest, grants no.911944). We would also thank statistician adjunct professor Geir Egil Eide, department of Global Public Health and Primary Care, Bergen University, Bergen Norway for this help in statistics analysis. 


\section{References:}

Ahlqvist KJ, Hamalainen RH, Yatsuga S, et al (2012) Somatic progenitor cell vulnerability to mitochondrial DNA mutagenesis underlies progeroid phenotypes in Polg mutator mice. Cell metabolism 15: 100-109.

Ahlqvist KJ, Leoncini S, Pecorelli A, et al (2015) MtDNA mutagenesis impairs elimination of mitochondria during erythroid maturation leading to enhanced erythrocyte destruction. Nature communications 6: 6494.

Antonicka H, Leary SC, Guercin GH, et al (2003) Mutations in COX10 result in a defect in mitochondrial heme A biosynthesis and account for multiple, early-onset clinical phenotypes associated with isolated COX deficiency. Human molecular genetics 12: 2693-2702.

Burrage LC, Tang S, Wang J, et al (2014) Mitochondrial myopathy, lactic acidosis, and sideroblastic anemia (MLASA) plus associated with a novel de novo mutation $(\mathrm{m} .8969 \mathrm{G}>\mathrm{A})$ in the mitochondrial encoded ATP6 gene. Molecular genetics and metabolism 113: 207-212.

Camaschella C (2009) Hereditary sideroblastic anemias: pathophysiology, diagnosis, and treatment. Seminars in hematology 46: 371-377.

Chen ML, Logan TD, Hochberg ML, et al (2009) Erythroid dysplasia, megaloblastic anemia, and impaired lymphopoiesis arising from mitochondrial dysfunction. Blood 114: 4045-4053.

Clarke SL, Bowron A, Gonzalez IL, et al (2013) Barth syndrome. Orphanet journal of rare diseases 8: 23.

Fontenay M, Cathelin S, Amiot M, Gyan E, Solary E (2006) Mitochondria in hematopoiesis and hematological diseases. Oncogene 25: 4757-4767.

Green DR, Kroemer G (2004) The pathophysiology of mitochondrial cell death. Science (New York, NY) 305: 626-629.

Hildick-Smith GJ, Cooney JD, Garone C, et al (2013) Macrocytic anemia and mitochondriopathy resulting from a defect in sideroflexin 4. American journal of human genetics 93: 906-914.

Hudson G, Chinnery PF (2006) Mitochondrial DNA polymerase-gamma and human disease. Human molecular genetics 15 Spec No 2: R244-252.

Li-Harms X, Milasta S, Lynch J, et al (2015) Mito-protective autophagy is impaired in erythroid cells of aged mtDNA-mutator mice. Blood 125: 162-174.

Lill R, Muhlenhoff U (2008) Maturation of iron-sulfur proteins in eukaryotes: mechanisms, connected processes, and diseases. Annual review of biochemistry 77: 669-700.

Lu YW, Claypool SM (2015) Disorders of phospholipid metabolism: an emerging class of mitochondrial disease due to defects in nuclear genes. Frontiers in genetics 6: 3 .

Morison IM, Cramer Borde EM, Cheesman EJ, et al (2008) A mutation of human cytochrome $\mathrm{c}$ enhances the intrinsic apoptotic pathway but causes only thrombocytopenia. Nature genetics 40: 387-389.

Nguyen KV, Sharief FS, Chan SS, Copeland WC, Naviaux RK (2006) Molecular diagnosis of Alpers syndrome. Journal of hepatology 45: 108-116.

Nordin G, Martensson A, Swolin B, et al (2004) A multicentre study of reference intervals for haemoglobin, basic blood cell counts and erythrocyte indices in the adult population of the Nordic countries. Scandinavian journal of clinical and laboratory investigation 64: 385-398.

Ojala T, Polinati P, Manninen T, et al (2012) New mutation of mitochondrial DNAJC19 causing dilated and noncompaction cardiomyopathy, anemia, ataxia, and male genital anomalies. Pediatric research 72: 432-437. 
Pearson HA, Lobel JS, Kocoshis SA, et al (1979) A new syndrome of refractory sideroblastic anemia with vacuolization of marrow precursors and exocrine pancreatic dysfunction. The Journal of pediatrics 95: 976-984.

Rensvold JW, Krautkramer KA, Dowell JA, Denu JM, Pagliarini DJ (2016) Iron Deprivation Induces Transcriptional Regulation of Mitochondrial Biogenesis. The Journal of biological chemistry.

Rensvold JW, Ong SE, Jeevananthan A, Carr SA, Mootha VK, Pagliarini DJ (2013) Complementary RNA and protein profiling identifies iron as a key regulator of mitochondrial biogenesis. Cell reports 3: 237-245.

Richardson DR, Lane DJ, Becker EM, et al (2010) Mitochondrial iron trafficking and the integration of iron metabolism between the mitochondrion and cytosol. Proceedings of the National Academy of Sciences of the United States of America 107: 10775-10782.

Riley LG, Rudinger-Thirion J, Schmitz-Abe K, et al (2016) LARS2 Variants Associated with Hydrops, Lactic Acidosis, Sideroblastic Anemia, and Multisystem Failure. JIMD reports 28: 49-57.

Rotig A, Bourgeron T, Chretien D, Rustin P, Munnich A (1995) Spectrum of mitochondrial DNA rearrangements in the Pearson marrow-pancreas syndrome. Human molecular genetics 4: 1327-1330.

Saneto RP, Naviaux RK (2010) Polymerase gamma disease through the ages. Developmental disabilities research reviews 16: 163-174.

Schmitz-Abe K, Ciesielski SJ, Schmidt PJ, et al (2015) Congenital sideroblastic anemia due to mutations in the mitochondrial HSP70 homologue HSPA9. Blood 126: 2734-2738.

Shteyer E, Saada A, Shaag A, et al (2009) Exocrine pancreatic insufficiency, dyserythropoeitic anemia, and calvarial hyperostosis are caused by a mutation in the COX4I2 gene. American journal of human genetics 84: 412-417.

Torraco A, Bianchi M, Verrigni D, et al (2017) A novel mutation in NDUFB11 unveils a new clinical phenotype associated with lactic acidosis and sideroblastic anemia. Clinical genetics 91: 441-447.

Trifunovic A, Wredenberg A, Falkenberg M, et al (2004) Premature ageing in mice expressing defective mitochondrial DNA polymerase. Nature 429: 417-423.

Tzoulis C, Engelsen BA, Telstad W, et al (2006) The spectrum of clinical disease caused by the A467T and W748S POLG mutations: a study of 26 cases. Brain : a journal of neurology 129: 1685-1692.

Wedatilake Y, Niazi R, Fassone E, et al (2016) TRNT1 deficiency: clinical, biochemical and molecular genetic features. Orphanet journal of rare diseases 11: 90.

Winterthun S, Ferrari G, He L, et al (2005) Autosomal recessive mitochondrial ataxic syndrome due to mitochondrial polymerase gamma mutations. Neurology 64: 12041208.

Wortmann SB, Zietkiewicz S, Kousi M, et al (2015) CLPB mutations cause 3methylglutaconic aciduria, progressive brain atrophy, intellectual disability, congenital neutropenia, cataracts, movement disorder. American journal of human genetics 96 : 245-257. 


\section{Figure legends}

Figure 1. Kaplan Meier curve comparing survival in those with and those without anaemia (total $=59$ ). No data was available for two patients. Number of events is given in parentheses.

Figure 2. Schematic diagram of the $P O L G$ gene illustrating the mutations identified in this study that were associated with anaemia (blue boxes indicate polymorphisms). More details are provided in supplementary file 1 . 
Table 1. Summary of the previously described haematological disorders due to mitochondrial dysfunction

\begin{tabular}{|c|c|}
\hline Disorder & Associated gene defect \\
\hline Pearson syndrome & Large deletion in mtDNA \\
\hline $\mathrm{X}$-linked sideroblastic anaemia & ALAS2 \\
\hline SLC5A38 related sideroblastic anaemia & SLC25A38 \\
\hline GLRX5-related sideroblastic anaemia & GLRX5 \\
\hline $\mathrm{X}$-linked sideroblastic anaemia with ataxia & $A B C B 7$ \\
\hline Macrocytic anaemia and mitochondrial disease & SFXN4 \\
\hline $\begin{array}{l}\text { Mitochondrial myopathy, lactic acidosis and sideroblastic anaemia } \\
\text { (MLASA) }\end{array}$ & PUS1, YARS2, MT-ATP6 \\
\hline $\begin{array}{l}\text { Congenital sideroblastic anaemia, B-cell immunodeficiency, periodic } \\
\text { fevers, and developmental delay (SIFD) }\end{array}$ & TRNT1 \\
\hline Barth syndrome & $T A Z$ \\
\hline Dilated cardiomyopathy with ataxia (DCMA) & DNAJC19 \\
\hline Friedreich ataxia & $F X N$ \\
\hline $\begin{array}{l}\text { Exocrine pancreatic insufficiency, dyserythropoeitic anemia, and } \\
\text { calvarial hyperostosis }\end{array}$ & COX4I2 \\
\hline Leigh syndrome and anaemia & COX10 \\
\hline Congenital thrombocytopenia & CYCS \\
\hline $\begin{array}{l}\text { Hydrops, lactic acidosis, sideroblastic anemia, and multisystem } \\
\text { failure }\end{array}$ & LARS2 \\
\hline $\begin{array}{l}\text { Cataract, neutropenia, epilepsy, movement disorder and 3- } \\
\text { methylglutaconic aciduria }\end{array}$ & $C L P B$ \\
\hline $\begin{array}{l}\text { Sideroblastic anaemia, lactic acidosis, microphthalmia and } \\
\text { histiocytoid cardiomyopathy }\end{array}$ & NDUFB11 \\
\hline Congenital sideroblastic anaemia & HSPA9 \\
\hline
\end{tabular}


Table 2: Number of patients with anaemia according to age group.

\begin{tabular}{|l|c|c|c|}
\hline \multicolumn{1}{|c|}{ Age (sex) } & $\begin{array}{l}\text { Number of patients } \\
\text { with anaemia }\end{array}$ & $\begin{array}{l}\text { Median (Range) } \\
\text { Haemoglobin value g/dl }\end{array}$ & $\begin{array}{l}\text { Normal Haemoglobin } \\
\text { value g/dl }\end{array}$ \\
\hline $30-59$ days & 1 & $7.0^{*}$ & $8.7-13.5$ \\
\hline $60-180$ days & 3 & $7.7(5.1-8.9)$ & $9.7-13.3$ \\
\hline 6 months -1 year & 5 & $8.0(6.1-10.0)$ & $10.3-13.2$ \\
\hline $1-5$ years & 8 & $8.2(7.2-10.2)$ & $10.6-13.8$ \\
\hline $6-11$ years & 2 & $9.6(9.3-10.0)$ & $10.6-14.4$ \\
\hline $12-17$ years (Female) & 2 & $8.5(7.2-9.9)$ & $10.7-16.5$ \\
\hline $12-17$ years (Male) & 1 & $8.8 *$ & $11.7-15.3$ \\
\hline$\geq 18$ years (Female) & 11 & $10.0(7.2-11.5)$ & $13.4-17.0$ \\
\hline$\geq 18$ years (Male) & 8 & $10.0(7.3-12.0)$ & \\
\hline
\end{tabular}

Median haemoglobin values with range for each age category are given. For those categories with a single patient $*=$ Actual haemoglobin. Normal reference values of haemoglobin for age and sex are given in the far right column. 
Figure 1

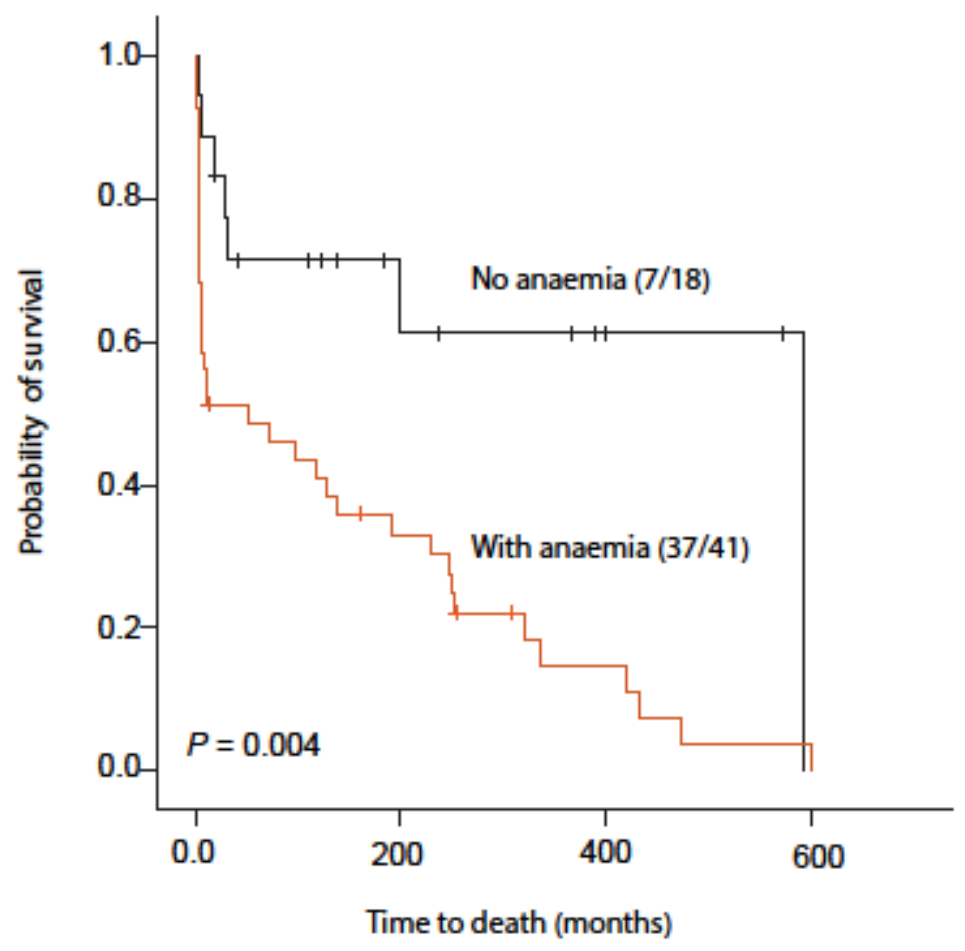

Figure 2 


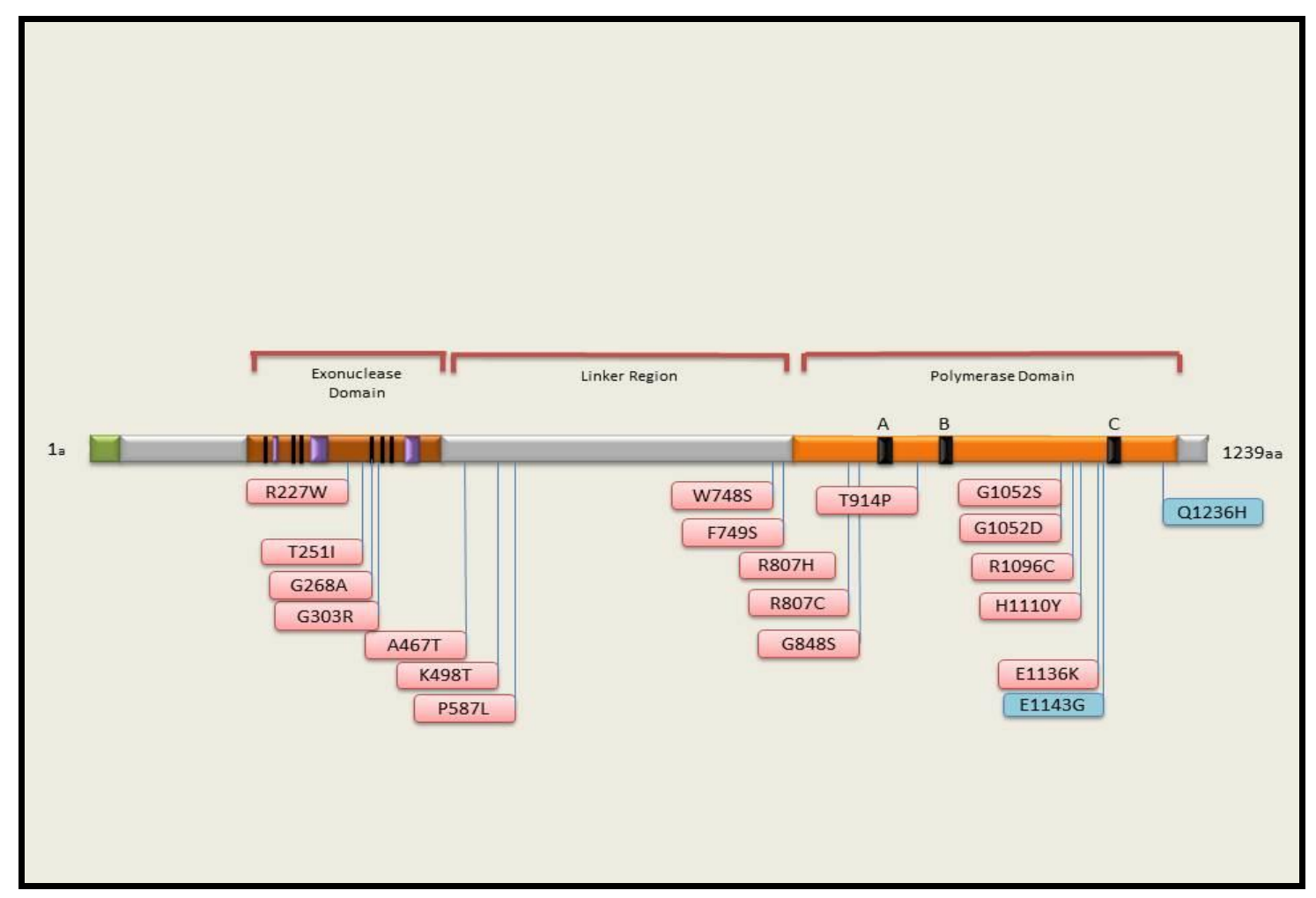

